\title{
Who's Who in the Society for International Development 1957 to 2007
}

\author{
Chairperson of the Society \\ Barbara Ward 1976-1981

\section{Advisor to the Society \\ 1989-1991} \\ HRH Prince Claus of the Netherlands
}

\section{President Emeritus}

Enrique Iglesias 1991-present

\section{Presidents}

Robert W. Hudgens 1957-1959

Hugh L. Keenleyside 1959

P.S.N. Prasad 1960

Jose'Antonio Mayobre 1961-1962

Paul G. Hoffman 1963-1965

S.O. Adebo 1966-1967

Dudley Seers 1968-1969

Felipe Herrera 1970-1971

Paul-Marc Henry 1972-1974

Irving S. Friedman 1975-1976

Sartaj Aziz 1976-1979

James P. Grant 1979-1982

Ismail Sabri Abdalla 1982-1985

Edgard Pisani 1985-1988

Enrique Iglesias 1988-1991

Maurice Williams 1991-1994

Nafis Sadik 1994-1997

Boutros Boutros-Ghali 1997-2000

Enrique Iglesias 2001-present

\section{Vice Presidents}

P.S.N. Prasad 1959

Henry VanZile Hyde, North America 1959-1960

Raul Prebisch, Latin America 1961

Arthur T. Mosher 1960

Henry VanZile Hyde 1961-1962

Luther H. Evans, North America 1961-1962

Felipe Herrera, Latin America 1962-1965

Ramon Binamira, Asia 1961-1962

Egbert deVries, Europe 1963-1965

K.S. Krishnaswamy, Asia 1963-1964

Michael L. Hoffman, North America 1963-1965

Robert K. Gardiner, Africa 1963-1965

K.B. Madhava, Asia 1964-1965

Marion Clawson 1963-1965

Robert E. Asher 1966-1967

Michael L. Hoffman 1968

Felipe Pazos 1969

Maurice Domergue 1970-1971

Irving S. Friedman 1972-1974

Sartaj Aziz 1975-1976

James P. Grant 1976-1979

\author{
Ismail Sabri Abdalla 1979-1982 \\ Mahbub ul Haq 1979-1982 \\ Richard Jolly 1982-1995 \\ Maurice Williams 1982-1985 \\ Marie-Angeliqué Savané 1985-1988 and 1994-1997 \\ Robert J. Berg 1985-1988 \\ QK. Ahmad 1988-1991 \\ Margaret Goodman 1988-1991 \\ Lourdes Arizpe 1991-1994 \\ Afaf M. Mahfouz 1991-1994 \\ J.C.J. Mohrmann 1991-1994 \\ L.P. Mureithi 1991-1994 \\ Rigoberta Menchú 1994-1997 \\ Thorvald Stoltenberg 1994-1997 \\ Gita Sen 1997-2000 \\ Jos van Gennip 1997-present \\ Khawar Mumtaz 2001-present
}

\section{Secretaries General}

Paul-Marc Henry 1977-1980

Ponna Wignaraja 1980-1986

Maurice Williams 1986-1991

Robert Cassani 1991-1992

Roberto Savio 1993-2001

\begin{abstract}
Executive Secretaries
Gove Hambidge 1957-1959

Robert L. Oshins 1959

Marion Clawson 1959-1962

Andrew E. Rice 1962-1977

Roberto Vanore 1978-1979

Henny Helmich 1986-1989

Robert Cassani 1989-1995

Stefano Prato 1995-present
\end{abstract}

\section{Officers of the NRST}

Chairs

Barbara Ward 1978-1980

Mahbub ul Haq 1980-1984

Maurice Strong 1984-1987

Richard Jolly 1987-1997

Khadija Haq 1997-present

\section{Editors in Chief of Development}

Andrew E. Rice 1959-1980

Ponna Wignaraja 1981-1986

Maurice Williams 1987-1997

Editors of Development

Ann Mattis 1981-1984

Wolfgang Sachs 1984-1986

Wendy Harcourt 1995-present

Development (2007) 50(S1), 160. doi:10.1057/palgrave.development.1100399 